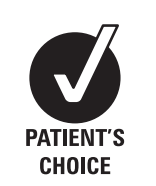

CHOICE

\title{
Integrated care improves risk-factor modification after stroke: initial results of the Integrated Care for the Reduction of Secondary Stroke model
}

\author{
J Joubert, ${ }^{1,2} \mathrm{C}$ Reid, ${ }^{3} \mathrm{D}$ Barton, ${ }^{4} \mathrm{~T}$ Cumming, ${ }^{2} \mathrm{~A}$ McLean, ${ }^{2} \mathrm{~L}$ Joubert, ${ }^{5} \mathrm{~J}$ Barlow, ${ }^{2}$ \\ D Ames, ${ }^{2} S$ Davis ${ }^{1}$
}

${ }^{1}$ Department of Neurology, Royal Melbourne Hospital, Melbourne, Australia; ${ }^{2}$ National Ageing Research Institute,

Parkville, Victoria, Australia;

${ }^{3}$ Department of Epidemiology and Preventive Medicine, Monash University, Melbourne, Australia; ${ }^{4}$ Department of Psychiatry, Royal Melbourne Hospital, Melbourne, Australia;

${ }^{5}$ School of Social Work, University of Melbourne, Melbourne, Australia

Correspondence to: Dr J Joubert, National Ageing Research Institute, 34-54 Poplar Road, Parkville, Victoria, Australia, 3052.

jacquesjoubert@bigpond.com

Received 26 February 2008 Revised 24 September 2008 Accepted 6 October 2008 Published Online First 14 November 2008

\section{ABSTRACT}

Objective: Despite evidence demonstrating that riskfactor management is effective in reducing recurrent cerebrovascular disease, there are very few structured care programmes for stroke survivors. The aim was to implement and evaluate an integrated care programme in stroke.

Methods: 186 patients with stroke were randomised to either the treatment (integrated care) or control (usual care) group and were followed up over 12 months. The Integrated Care for the Reduction of Secondary Stroke (ICARUSS) model of integrated care involved collaboration between a specialist stroke service, a hospital coordinator and a patient's general practitioner. The primary aim was to promote the management of vascular risk factors through ongoing patient contact and education.

Results: In the 12 months poststroke, systolic blood pressure (sBP) decreased in the treatment group but increased in controls. The group difference was significant, and remained so when age, sex, disability and sBP at discharge were accounted for $(p=0.04)$. Treatment patients also exhibited better modification of body mass index ( $p=0.007)$ and number of walks taken $(p<0.001)$ than controls. Rankin scores indicated significantly reduced disability in treatment patients relative to controls in the year poststroke $(p=0.003)$.

Conclusions: Through an integrated system of education, advice and support to both patient and GP, the ICARUSS model was effective in modifying a variety of vascular risk factors and therefore should decrease the likelihood or recurrent stroke or vascular event.

Stroke recurrence is a consistent and independent predictor of disability, institutionalisation and death, often resulting in a stepwise decline into dependency in stroke survivors. ${ }^{1}$ The burden of stroke is expected to increase in future years with the rapid rise in older people and the decline in stroke mortality. ${ }^{2}$ Secondary prevention of stroke, therefore, is of paramount importance. It has been estimated that the successful management of recognised vascular risk factors can reduce stroke incidence by $70-80 \% .^{3-5}$

Yet despite the wide availability of published guidelines, consensus statements and directives, ${ }^{67}$ implementation of evidence-based recommendations is often suboptimal in both the hospital setting and after discharge. ${ }^{8-1028-30}$ The reasons for this are complex. Absence of recommended hospital protocols, busy General Pratitioner (GP) practices with short consultation times and a wide range of conditions to contend with, non-availability of ready advice for the GP and lack of clear, practical guidelines for the GP regarding risk-factor management are some of the reasons.

The successful adoption of these recommendations has major health and economic implications. ${ }^{11}$ In the last decade, several programmes and strategies promoting prevention of recurrent stroke have been trialled, both within hospital and after discharge. ${ }^{12-14}$ Altering physician and patient behaviour patterns is difficult, and achieving long-term changes in stroke prevention care is complex. ${ }^{15}$

The Integrated Care for the Reduction of Secondary Stroke (ICARUSS) model is a novel and multimodal programme aimed at facilitating the implementation of recommended stroke prevention strategies. The model incorporates a "shared care" component, which has been effective in improving the long-term management of several chronic diseases ${ }^{16}$ but has not previously been applied to stroke. We report results from a randomised controlled trial evaluating the effect of this programme on risk-factor modification, lifestyle changes, patient education and disability in a cohort of stroke survivors returning to their primary care physicians.

\section{METHODS}

\section{Development of the ICARUSS model}

The ICARUSS (IC) model was developed after extensive consultation with representatives from General Practice in Melbourne, Australia. It addresses risk factors for both ischaemic stroke and parenchymal haemorrhage. The goals and recommendations were derived from national directives, evidence-based guidelines and consensus statements regarding risk-factor management. A clinical coordinator promotes patient education and the bidirectional flow of clinically important information between stroke specialists and primary care physicians. Exposure to the model is initiated during the acute in-hospital phase and continued after discharge, thereby combining both the early implementation of risk management strategies and long-term risk reduction. The IC model includes an aspect of shared care, whereby both specialist services and primary-care physicians take "contemporaneous responsibility" for the ongoing management of patients. The GP has ready telephonic access at all times to a stroke specialist for advice.

The ICARUSS protocol targets seven modifiable risk factors: blood pressure, cholesterol, atrial fibrillation, body mass index (BMI), smoking, 
Table 1 Patient characteristics for the integrated care (IC) and standard care (SC) groups

\begin{tabular}{lcll}
\hline & IC (n= 91) & SC $(\mathbf{n}=\mathbf{9 5})$ & Significance \\
\hline Sex (male) & $53(58 \%)$ & $49(52 \%)$ & $\chi^{2}=0.8, \mathrm{NS}$ \\
Age (SD) & $63.4(13.7)$ & $68.2(12.7)$ & $\mathrm{t}(183)=2.5, \mathrm{p}=0.015$ \\
Stroke type-infarct & $66(73 \%)$ & $76(80 \%)$ & \\
$\quad$ Haemorrhage & $9(10 \%)$ & $7(7 \%)$ & \\
$\quad$ Transient ischaemic attack & $15(17 \%)$ & $12(13 \%)$ & $\chi^{2}=1.2, \mathrm{NS}$ \\
Lesion side-left & $47(52 \%)$ & $38(41 \%)$ & \\
$\quad$ Right & $37(41 \%)$ & $46(49 \%)$ & \\
$\quad$ Bilateral & $7(8 \%)$ & $9(10 \%)$ & $\chi^{2}=2.2, \mathrm{NS}$ \\
\hline
\end{tabular}

alcohol intake and physical activity. To address these, the main interventions include the early prescription of appropriate medications (antithrombotics, anticoagulants, diabetic treatment, antihypertensives and cholesterol-lowering statins) by the medical staff, and promotion of lifestyle changes (smoking cessation, reduction in alcohol intake, maintenance of recommended BMI and deliberate physical exercise) by the nurse coordinator. Important goals were the early in-hospital initiation and sustained use of appropriate medical treatment and the early commencement of education by the nurse coordinator regarding behaviour modification. In addition, the programme provided education to patients and carers regarding stroke risk factors and the warning signs for stroke. Periodic telephone screening for depressive symptoms by the study coordinator was incorporated into the structure of the IC model. Bestpractice management was instituted in hospital by the stroke neurologists, and further management in the community was decided on by the treating primary care physicians (guided by the goals and recommendations supplied to them in the ICARUSS flow charts). Further details of interventions are documented in the sections below under "predischarge procedure" and postdischarge procedure."

\section{Objectives}

The central objective was to promote the early initiation and long-term maintenance of best-practice recommendations for risk-factor management in stroke survivors returning to the care of their GPs. The primary outcome measure was blood pressure. Secondary outcome measures were: cholesterol levels, atrial fibrillation, smoking, alcohol intake, weight and physical activity (deliberate exercise walks). We hypothesised that patients exposed to the IC model would exhibit better management of vascular risk factors and receive more targeted risk-factor advice than patients receiving standard care. A second objective was to investigate the effect of the IC model on disability, activities of daily living, cognitive function and quality of life.

\section{Inclusion and exclusion criteria}

All patients who were aged 20 years and older and who were admitted between 2000 and 2004 to the Royal Melbourne Hospital or Western General Hospital with transient ischaemic attack (TIA) or completed stroke (cerebral infarction or parenchymal haemorrhage), as confirmed by CT scan, were considered for inclusion. The study received approval from the relevant Ethics Committees at the Royal Melbourne and Western Hospitals. Patients were excluded if they: (1) were not returning to their GPs for management, (2) were discharged to a nursing home, (3) had serious comorbidities, (4) were nonEnglish-speaking, (5) died while in hospital, (6) were too cognitively impaired, (7) were notably aphasic or (8) lived more than $2 \mathrm{~h}$ away by car or (9) suffered from subarachnoid haemorrhage or subdural haematoma. Other reasons for nonparticipation included the family declining to take part, involvement in another research programme and not being assessed prior to discharge. The study coordinator enrolled and randomly assigned patients, according to a computer-generated process, to either the IC or SC group. Informed consent was obtained from all participating patients. All subjects were clinically evaluated by a stroke neurologist and had computerised tomography (CT) or MRI of the brain and routine blood evaluations (including lipid profiles). Where appropriate, other investigations such as chest radiographs and transoesophageal echo (TOE) were carried out.

The allocation to group was undertaken after consent, so the coordinator was unaware of treatment allocation prior to consent. After a patient had agreed to participate in the study, informed consent was obtained from them. After this had

Table 2 Mean risk-factor measures at discharge and 12 months, and mean change scores, for the integrated care (IC) and standard care (SC) groups (standard deviations in parentheses)

\begin{tabular}{llccrcc}
\hline & Group & Discharge & 12 months & Change & t Test & ANCOVA \\
\hline sBP & IC & $134.2(17.0)$ & $128.5(13.7)$ & $6.0(20.1)$ & & \\
dBP & SC & $131.2(19.2)$ & $134.5(19.4)$ & $-1.8(24.2)$ & $\mathrm{p}=0.02$ & $\mathrm{p}=0.04$ \\
& IC & $76.1(11.7)$ & $77.3(8.3)$ & $-1.0(12.3)$ & & \\
Cholesterol & SC & $75.6(12.0)$ & $79.1(8.9)$ & $-2.8(13.0)$ & $\mathrm{p}=0.37$ & \\
\multirow{3}{*}{ Body mass index } & IC & $5.1(1.0)$ & $4.9(1.0)$ & $0.3(1.0)$ & & \\
\multirow{2}{*}{ Walks } & SC & $5.2(1.4)$ & $5.0(1.0)$ & $0.2(1.4)$ & $\mathrm{p}=0.74$ & \\
& IC & $28.1(5.8)$ & $27.5(5.4)$ & $0.5(2.1)$ & & \\
& SC & $28.1(5.6)$ & $28.7(6.3)$ & $-0.3(2.4)$ & $\mathrm{p}=0.04$ & $\mathrm{p}=0.007$ \\
\hline
\end{tabular}

Cholesterol, total cholesterol; dBP, diastolic blood pressure; sBP, systolic blood pressure; Walks, deliberate exercise walks per week. 
Table 3 Comparison of the number of integrated care (IC) and standard care (SC) patients with categorical risk factors at discharge and 12 months

\begin{tabular}{llrrr}
\hline & Group & Discharge & 12 months & 12-month difference \\
\hline Atrial fibrillation & IC & $13 / 86(15 \%)$ & $12 / 82(15 \%)$ & \\
\multirow{2}{*}{ Smoking } & SC & $18 / 86(21 \%)$ & $20 / 85(24 \%)$ & $\chi^{2}=2.1$, NS \\
& IC & $13 / 89(15 \%)$ & $14 / 91(15 \%)$ & \\
Alcohol $(>1$ drink per day) & SC & $9 / 90(10 \%)$ & $10 / 92(11 \%)$ & $\chi^{2}=0.8$, NS \\
& IC & $22 / 91(24 \%)$ & $13 / 91(14 \%)$ & \\
& SC & $18 / 95(19 \%)$ & $21 / 95(22 \%)$ & $\chi^{2}=1.9, N S$ \\
\hline
\end{tabular}

occurred, the randomisation schedule on the computer was checked, and the patient was randomly assigned to one of the two groups. At a later stage, the coordinator checked the patient's GP, and if this GP was also responsible for a different patient already in the trial, the current patient was assigned the same group as this previous patient.

\section{Predischarge procedure}

Prior to discharge from hospital, a final clinical diagnosis was obtained on all patients according to the TOAST classification ${ }^{17}$ and a detailed risk-factor profile was recorded for each patient. All patients were evaluated on a range of neurological, radiological and clinical measures. Clinical assessments included the Rankin scale, ${ }^{18}$ used to measure physical disability, the Barthel index, ${ }^{19}$ used to evaluate impairments in activities of daily life, the Mini-Mental State Examination (MMSE), ${ }^{20}$ used to assess cognitive function, and the Assessment of Quality-ofLife Questionnaire (AQoL). ${ }^{21}$

\section{IC group}

For patients randomised to the IC group, the role of the study coordinator and the goal of telephone tracking by the coordinator were explained. Education was given regarding the importance of the effective management of modifiable risk factors. Visits were prearranged with the GP's office for 2 weeks, 3 months, 6 months, 9 months and 12 months postdischarge. If appropriate, a carer was identified.

\section{SC group}

For patients in the SC group, arrangements were made for the study coordinator to contact them in 12 months for evaluation.

\section{Postdischarge procedure}

IC group

\section{GP documentation and tools}

GPs were sent an explanatory letter detailing the shared care process, and a typed discharge summary detailing relevant investigations, risk-factor measures, medication and planned management. They also received a flow chart containing goals and recommendations for risk-factor management based on published evidence-based guidelines. This flow chart included space for risk-factor data and other documentation to be entered at each scheduled GP visit.

\section{Telephone tracking}

Prior to each 3-monthly scheduled GP visit, the study coordinator conducted a semistructured telephone interview with the patient or carer, or both. Information about current general problems experienced by patient or carer, the number of walks taken, the number of cigarettes smoked and quantity of alcohol consumed per week was obtained. Finally, a screening tool for depression, validated for telephone administration, was administered. This information was faxed to the GP before each scheduled consultation.

\section{Follow-up procedures}

After each GP visit, the patient or carer was telephoned to check whether the visit took place and if there were any changes to medication or management. The relevant risk-factor data and medications prescribed, which had been documented by the GP at the visit and faxed back to the coordinator, were entered into a database and scrutinised by the coordinator. In the case of best-practice recommendations persistently not being met, the GP was contacted to discuss treatment review options.

Table 4 Percentage of integrated care (IC) and standard care (SC) patients who recalled receiving advice on certain risk factors, and percentage responding "yes" to other medical factors

\begin{tabular}{lccrc}
\hline & IC (\%) & SC (\%) & $\chi^{2}$ & Significance \\
\hline Risk factors & & & & \\
$\quad$ Blood pressure management & 80 & 64 & 6.1 & $\mathrm{p}=0.013$ \\
$\quad$ Cholesterol management & 81 & 51 & 19.5 & $\mathrm{p}<0.001$ \\
Smoking & 67 & 75 & 0.4 & $\mathrm{p}=0.55$ \\
Alcohol intake & 56 & 37 & 5.2 & $\mathrm{p}=0.023$ \\
$\quad$ Salt intake/weight & 54 & 50 & 0.2 & $\mathrm{p}=0.52$ \\
$\quad$ Physical activity & 81 & 63 & 7.9 & $\mathrm{p}=0.005$ \\
$\quad$ Blood glucose/diabetes & 78 & 63 & 4.7 & $\mathrm{p}=0.031$ \\
Other factors & & & & \\
$\quad$ Do you know the symptoms of transient ischaemic attack? & 61 & 31 & 15.9 & $\mathrm{p}<0.001$ \\
$\quad$ Have you been advised to seek immediate medical help in case of transient & 74 & 50 & 11.0 & $\mathrm{p}=0.001$ \\
ischaemic attack? & & & & \\
$\quad$ Do you have regular blood tests? & 91 & 73 & 10.5 & $\mathrm{p}=0.001$ \\
\hline
\end{tabular}


Table 5 Mean scores at discharge and 12 months, and mean change scores, for integrated care (IC) and standard care (SC) groups (standard deviations in parentheses)

\begin{tabular}{llccccc}
\hline & Group & Discharge & 12 months & Change & t Test & ANCOVA \\
\hline Rankin & IC & $1.7(1.2)$ & $1.2(1.1)$ & $0.4(1.0)$ & & \\
& SC & $1.9(1.3)$ & $1.9(1.2)$ & $0.0(1.3)$ & $\mathrm{p}=0.012$ & $\mathrm{p}=0.003$ \\
Barthel & IC & $17.8(3.6)$ & $19.1(2.2)$ & $1.3(3.4)$ & & \\
& SC & $16.8(4.7)$ & $17.8(3.8)$ & $1.0(4.2)$ & $\mathrm{p}=0.64$ & \\
MMSE & IC & $20.5(4.6)$ & $21.0(4.0)$ & $0.5(4.0)$ & & \\
\multirow{2}{*}{ AOoL } & SC & $18.6(5.8)$ & $19.0(5.2)$ & $0.5(4.4)$ & $\mathrm{p}=0.97$ & \\
& IC & $26.0(6.8)$ & $26.4(5.3)$ & $-0.3(5.0)$ & & \\
& SC & $26.7(6.1)$ & $29.7(6.2)$ & $-2.9(5.2)$ & $\mathrm{p}=0.002$ & $\mathrm{p}=0.012$ \\
\hline
\end{tabular}

$\mathrm{A} 0 \mathrm{~L}$, assessment of quality-of-life questionnaire; Barthel, Barthel index; MMSE, mini-mental state examination; Rankin, Rankin scale.

\section{SC group}

SC patients were discharged to standard care from their GP. The frequency of visits, the guidelines adopted and the actions taken were left to the discretion of the GP.

\section{Evaluation at 12 months}

All patients were evaluated at 12 months with respect to riskfactor management. The neurological and clinical assessments that were given at discharge were repeated at 12 months. Patients also underwent a structured interview regarding the educational information on vascular risk factors they received over the 12 months, as well as their knowledge of warning signs for stroke and TIA.

\section{Statistical analysis}

For continuous variables, within-subject changes were expressed as differences between baseline (discharge) and 12month values. Independent $t$ tests were used to analyse the between-group differences in change scores. In addition, ANCOVAs were conducted to test whether these differences remained when baseline values and other variables-age, sex and Rankin at discharge-were entered as covariates. Categorical data were analysed using descriptive and $\chi^{2}$ statistics. To evaluate the predictors of disability at 12 months, both univariate and multivariate logistic regressions were conducted. Patients were classified as either disabled (Rankin $>2$ ) or non-disabled (Rankin 0-2). The variables of group, age, sex and cognitive function (MMSE at 12 months) were included in the analyses. Any variable that had a significance of $p<0.20$ in univariate regression was included in multivariate regression, and all variables with a significance of $\mathrm{p}<0.10$ combined to form the final model. Inevitably, there were some missing values, but these were mostly in the IC group from the midyear follow-ups (3, 6 or 9 months) and data from these times do not feature in the current analyses. All analyses were conducted using SPSS statistical software, and $p$ values of less than 0.05 were reported as significant.

\section{RESULTS \\ Demographics and clinical features}

Between February 2000 and September 2004, a total of 233 patients with stroke were enrolled into the study. About 500 patients with cerebrovascular disease who were discharged to a nursing home were not eligible for the study. Of the 233 patients, 123 were randomised to the IC group and 110 to the SC group. Forty-seven patients were lost to follow-up, with reasons including: patient unwilling to participate, patient experiencing other medical problems, patient did not have a stroke, patient changed GP, patient not contactable and patient deceased. For full details, see the participant flow chart in fig 1. There were 14 GPs who had two participants enrolled in the trial. In 12 of the 14 cases, the second participant was allocated to the same group as the initial participant in order to avoid contamination. The majority of these 12 happened to be allocated to the intervention group, which led to a slight imbalance in treatment allocation. The other two of the 14 cases were inadvertently missed, resulting in two GPs each being responsible for two patients who were not in the same treatment group.

Overall, a total of 186 stroke survivors were included in the study: 91 in the IC group and 95 in SC. To avoid contamination, if more than one patient was treated by the same GP, all subsequent patients were allocated to the same group as the first patient to prevent contamination of the sample.

\section{Risk-factor modification}

Demographic information for the treatment and control groups is shown in table 1.

Risk factors were measured at discharge and 12 months, and from these measures, 12 -month change scores were calculated (see table 2).

IC group patients were significantly more successful in lowering their sBP, reducing their BMI and increasing their walking than SC patients, even when covariates were accounted for. For sBP, a 12-month target was set of less than $140 \mathrm{~mm} \mathrm{Hg}$, and this was reached by $66 / 88$ (75\%) of the IC group but only $52 / 90(58 \%)$ of the SC group $\left(\chi^{2}=5.9, p=0.015\right)$. The recommended target level for total cholesterol was less than $5.18 \mathrm{mmol} / \mathrm{l}$, and this was attained by $64 \%$ of IC group patients but only $55 \%$ of SC patients $\left(\chi^{2}=1.4\right.$, NS). Other risk factorsatrial fibrillation, smoking and alcohol intake-were analysed categorically. The number of subjects with atrial fibrillation, smoking and drinking $>1$ standard drink per day both at discharge and at 12 months was not significantly different between the two groups (see table 3).

Of those with atrial fibrillation, more IC patients than SC patients were taking warfarin at 12 months $(10 / 12$ (83\%) vs $13 /$ $\left.20(65 \%) ; \chi^{2}=1.2, \mathrm{NS}\right)$.

More IC patients than SC patients remembered receiving advice on various risk factors (see table 4).

The group differences were significant for advice pertaining to blood pressure, cholesterol, alcohol intake, physical activity and diabetes. There was some evidence that this advice translated into risk-factor modification. For those receiving advice on cholesterol management, the average cholesterol reduction was $0.47 \mathrm{mmol} / \mathrm{l}$, whereas without advice there was an average gain of $0.15 \mathrm{mmol} / \mathrm{l}$. This difference in cholesterol change scores was significant $(t(130)=2.8, p=0.005)$. 
Figure 1 Participant flow chart, summarising patient numbers and reasons for exclusion. IC, integrated care; SC, standard care.

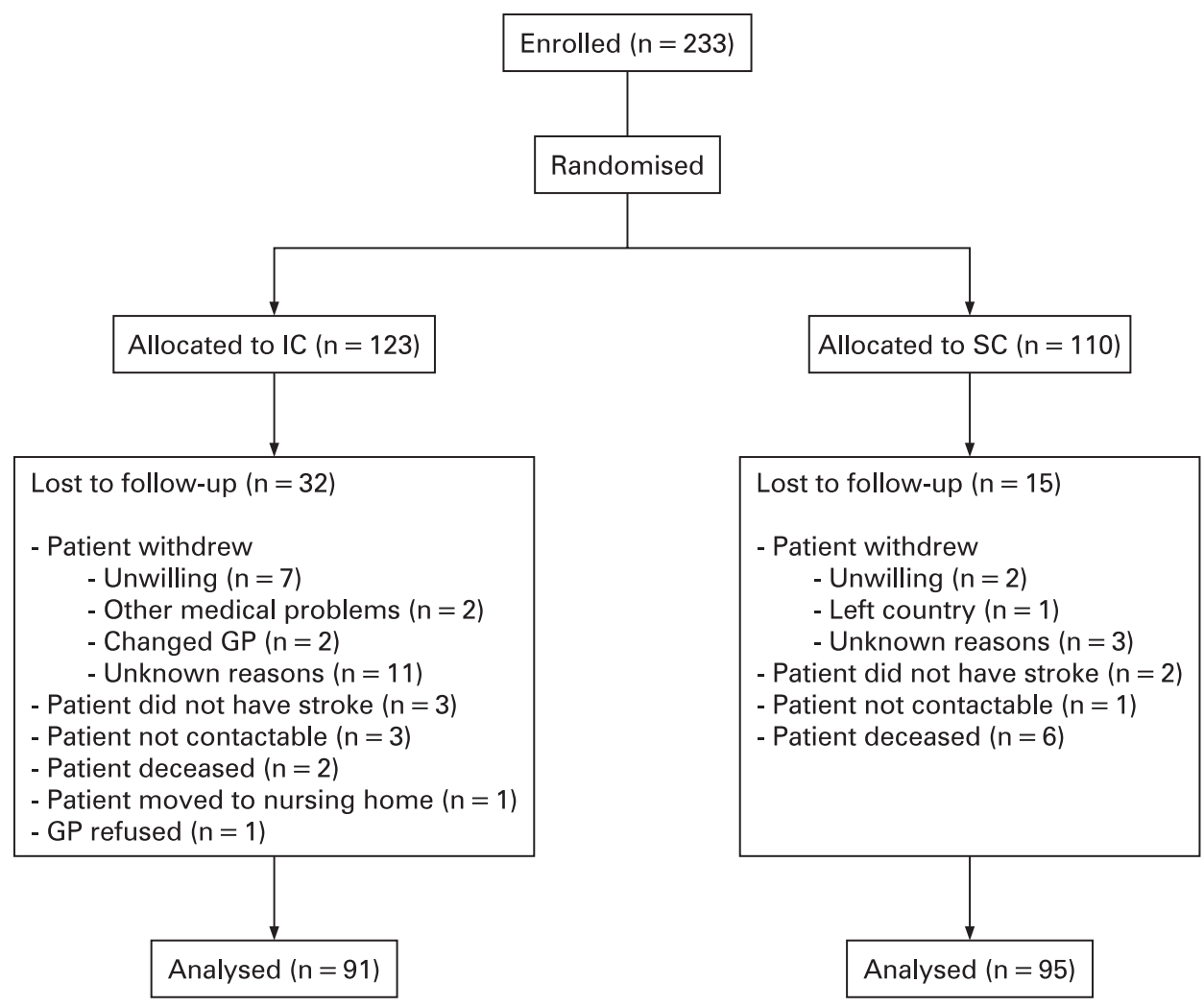

\section{Clinical outcomes}

Results from assessments of disability, activities of daily living, cognitive function and quality of life are presented in table 5 .

The group differences in 12-month change on the Rankin scale and $\mathrm{AQoL}$ questionnaire remained significant when relevant covariates were accounted for. At 12 months, only $14 \%$ of IC patients were classed as disabled, compared with $33 \%$ of SC controls $\left(\chi^{2}=8.5, p=0.003\right)$. Univariate logistic regression indicated that group, age and MMSE at 12 months were all associated with being disabled at 12 months. All three variables remained as independent associates when included in a multivariate model: group $(B=0.79 ; \quad S E=0.41 ; p=0.056)$, age $(B=0.05 ; \quad S E=0.02 ; \quad p=0.010) \quad$ and 12-month MMSE $(\mathrm{B}=-.18 ; \mathrm{SE}=0.04 ; \mathrm{p}<0.001)$.

\section{DISCUSSION}

These pilot results demonstrate that the ICARUSS integrated care (IC) model, a multimodal programme that includes a shared care component, can have a positive long-term effect on risk-factor modification. Over 12 months, stroke and TIA survivors randomised to the IC group exhibited a significantly greater reduction in systolic blood pressure (sBP) and BMI than controls, and increased physical activity relative to controls. Furthermore, IC patients showed greater improvement in disability over the 12 months than controls. To our knowledge, this is the first randomised, multicentre trial that has demonstrated benefit in both traditional and lifestyle risk factors for cerebrovascular disease through the implementation of an integrated model of care.

Lowering blood pressure in stroke survivors can reduce the risk for both first and recurrent stroke by approximately $40 \%,{ }^{22}{ }^{23}$ underscoring the importance of sBP in stroke genesis. In this study, IC patients had an average 12-month decrease in sBP of $6 \mathrm{~mm} \mathrm{Hg}$, whereas the sBP of SC patients increased. In the JNC 7 Report, ${ }^{24}$ weight loss and regular physical activity were associated with sBP reduction. A reduction in risk of stroke from even moderate physical activity has been demonstrated.' Our results revealed an increase in the number of deliberate exercise walks taken over the 12 months poststroke in the IC group, but a decrease in the exercise walking of SC patients. IC patients also demonstrated more success in lowering their BMI than SC patients, whose weight increased in the year poststroke. These findings indicate that an integrated model of care can modify behaviour-related variables that have recently been identified as important risk factors for stroke. ${ }^{1125}$

Clinically relevant goals were set for sBP and cholesterol levels. Significantly more IC patients than controls attained the target sBP of less than $140 \mathrm{~mm} \mathrm{Hg}$ at 12 months. This finding is particularly notable, given that fewer IC patients than SC patients met this sBP target at discharge. Although there was no significant group difference in the reduction in serum cholesterol, $64 \%$ of IC patients attained a level of less than $5.18 \mathrm{mmol} / \mathrm{l}$ at $12 \mathrm{months}$, whereas this target was reached by only $55 \%$ of SC patients. Significantly more IC patients $(80 \%)$ than SC patients $(50 \%)$ recalled receiving advice on cholesterol management. Patients who received this advice made a significantly greater reduction in their cholesterol level than patients who did not receive advice.

Exposure to the IC model generated improvements in two of the clinical assessments. There was a decrease in the disability of IC patients over the 12 months poststroke, while disability in the SC group was stable. This difference was both statistically significant and clinically relevant: with scores on the Rankin scale dichotomised into "good" (0-2) and "bad" (3-5) outcome, only $14 \%$ of the IC patients had a bad outcome at 12 months, compared with $33 \%$ of SC patients. Health-related quality of life, as measured by the AQoL, remained constant over the 12 months in the IC group but decreased substantially in the SC 
group. The significant group differences in physical disability and quality of life remained after potentially confounding variables were accounted for in multivariate analyses.

Two shortcomings of this study need to be considered when interpreting the results. First, the IC patients were younger than the SC patients, and this may have influenced some of the group differences that were identified, that is with anticoagulation for AF and physical activity. Similarly, the differences in the modified Rankin scores may be explained by this. However, it is unlikely that age alone can account for the current set of results, and the reasons for these findings will be addressed in a follow-up study. Second, a systematic bias in the study relates to patients who were lost to follow-up (fig 1 )

All significant group differences in risk factors and clinical outcomes were confirmed using ANCOVAs that partialled out the effect of age, sex and other relevant variables. Second, discharge values for several risk factors and clinical measures were unexpectedly dissimilar in the IC and SC groups. Differing baselines can cause problems in the interpretation of change scores. This issue, too, was addressed by the inclusion of the relevant variable at discharge in the ANCOVA analyses.

Models for ensuring effective, long-term risk-factor management of stroke survivors have been elusive. A review of complex interventions in stroke care indicated that few have been either adequately designed or properly evaluated. ${ }^{15}$ The efficacy of the IC model can be attributed, at least in part, to techniques that have been successful in improving risk-factor management: telephone tracking and feedback, ${ }^{26}$ furnishing doctors with evidence-based guidelines, ${ }^{27}$ and putting in place point-of-care reminders. ${ }^{28}$ The model aims to correct recognised inadequacies in standard care: poor patient knowledge about risk factors after a stroke event, ${ }^{29}$ lack of systematic risk assessment in hospital, ${ }^{30}$ doctors' unfamiliarity or disagreement with guidelines ${ }^{28}$ and neurologists who do not consider risk-factor modification their responsibility. ${ }^{30}$ The flow chart supplied to the GP functions in a similar fashion to the standardised order sets described in the CASPR study. ${ }^{13}$ Based on current expert consensus, the charts are available at the point of care, and they are eminently suitable to changing guidelines for stroke care. There is no need for time-consuming educational sessions for GPs, and the information is up to date and accessible. Like PROTECT, ${ }^{12}$ the IC model commences secondary prevention in hospital. ICARUSS is unique, however, in that it provides patient and carer support and education, ongoing surveillance of risk factors, a bidirectional information feedback loop utilising cost-effective communication tools such as telephone-tracking and faxing, and specialist telephone support for the GP. Future work will evaluate the sustainability and transferability of the IC model, its applicability in different socio-economic, cultural and geographical settings, and its long-term effect on prevention of stroke.

Acknowledgements: This research was funded by a Commonwealth of Australia General Practice Evaluation Program grant.

\section{Competing interests: None.}

Ethics approval: Ethics approval was provided by the Ethics Committees at the Royal Melbourne and Western Hospitals.

Patient consent: Obtained.

\section{REFERENCES}

1. Hankey GJ, Jamrozik K, Broadhurst RJ, et al. Long-term disability after first-ever stroke and related prognostic factors in the Perth Community Stroke Study, 19891990. Stroke 2002;33:1034-40.

2. Muntner P, Garrett E, Klag MJ, et al. Trends in stroke prevalence between 1973 and 1991 in the US population 25 to 74 years of age. Stroke 2002;33:1209-13.

3. Hachinski V. Stroke: The next 30 years. Stroke 2002;33:1-4.

4. Yusuf S. Two decades of progress in preventing vascular disease. Lancet 2002;360:2-3.

5. Wald NJ, Law MR. A strategy to reduce cardiovascular disease by more than $80 \%$. BMJ 2003:326:1419-23.

6. Sacco RL, Adams R, Albers G, et al. Guidelines for prevention of stroke in patients with ischemic stroke or transient ischemic attack: A statement for healthcare professionals from the American Heart Association/American Stroke Association Council on Stroke. Stroke 2006;37:577-617.

7. Gorelick PB, Sacco RL, Smith DB, et al. Prevention of a first stroke: A review of guidelines and a multidisciplinary consensus statment from the National Stroke Association. JAMA 1999;281:1112-20.

8. Gibbs RGJ, Newson R, Lawrenson R, et al. Diagnosis and initial management of stroke and transient ischemic attack across UK health regions from 1992 to 1996. Stroke 2001;32:1085-90.

9. Goldstein LB, Farmer A, Matchar DB. Primary care physician-reported secondary and tertiary stroke prevention practices: A comparison between the United States and the United Kingdom. Stroke 1997;28:746-51.

10. Mouradian M, Majumdar SR, Senthilselvan A, et al. How well are hypertension, hyperlipidemia, diabetes, and smoking managed after a stroke or transient ischemic attack? Stroke 2002;33:1656-9.

11. Sanossian N, Ovbiagele B. Multimodality stroke prevention. Neurologist 2006;12:14-31.

12. Ovbiagele B, Saver JL, Fredieu A, et al. In-hospital initiation of secondary stroke prevention therapies yields high rates of adherence at follow-up. Stroke 2004;35:2879-83.

13. California Acute Stroke Pilot Registry (CASPR) Investigators. The impact of standardised stroke orders on adherence to best practices. Neurology 2005;65:360-5.

14. Smaha LA. The American Heart Association Get With The Guidelines program. Am Heart J 2004;148:46-8S.

15. Redfern J, McKevitt C, Wolfe CDA. Development of complex interventions in stroke care: A systematic review. Stroke 2006;37:2410-19.

16. Orton P. Shared care. Lancet 1994;344:1413-15.

17. Adams HP, Bendixen BH, Kappelle LJ, et al. Classification of subtype of acute ischemic stroke: Definitions for use in a multicenter clinical trial. Stroke 1993;24:35-41.

18. Bonita R, Beaglehole R. Modification of Rankin Scale: Recovery of motor function after stroke. Stroke 1988;19:1497-500.

19. Mahoney F, Barthel D. Functional Evaluation: the Barthel Index. Md Med J 1965;14:61-5.

20. Folstein MF, Folstein SE, McHugh PR. Mini-mental state: A practical method for grading the cognitive state of patients for the clinician. J Psychiatr Res 1975;12:189-98.

21. Hawthorne G, Richardson J, Osborne R. The Assessment of Quality of Life (AOOL) instrument: A psychometric measure of health-related quality of life. Qual Life Res 1999;8:209-24.

22. PROGRESS Collaborative Group. Randomised trial of a perindopril-based bloodpressure-lowering regimen among 6105 individuals with previous stroke or transient ischaemic attack. Lancet 2001;358:1033-41.

23. SHEP Cooperative Research Group. Prevention of stroke by antihypertensive drug treatment in older persons with isolated systolic hypertension: Final results of the Systolic Hypertension in the Elderly Program (SHEP). JAMA 1991;265:3255-64.

24. Chobanian AV, Bakris GL, Black HR, et al. The seventh report of the joint national committee on prevention, detection, evaluation, and treatment of high blood pressure. JAMA 2003;289:2560-72.

25. Sacco RL. Newer risk factors for stroke. Neurology 2001;57:31-4S.

26. Friedman RH, Kazis LE, Jette A, et al. A telecommunications system for monitoring and counseling patients with hypertension: Impact on medication adherence and blood pressure control. Am J Hypertens 1996;9:285-92.

27. Joseph LN, Babikian VL, Allen NC, et al. Risk factor modification in stroke prevention-The experience of a stroke clinic. Stroke 1999;30:16-20.

28. Ornstein SM. Translating research into practice using electronic medical records. The PPRNet-TRIP project: Primary and secondary prevention of coronary heart disease and stroke. Top Health Inf Manage 2002;22:52-8.

29. Travis LH, Flemming KD, Brown RD Jr, et al. Awareness of stroke risk factors, symptoms and treatment is poor in people at highest risk. J Stroke Cerebrovasc Dis 2003:12:221-7.

30. Holloway RG, Benesch C, Rush SR. Stroke prevention: Narrowing the evidencepractice gap. Neurology 2000;54:1899-906. 